\title{
HARMONICITY UP TO REARRANGEMENT AND ISOTHERMAL GAS DYNAMICS*
}

\author{
YANN BRENIER ${ }^{\dagger}$
}

Abstract. The concept of harmonic (or wave) maps up to rearrangement is introduced. A relation is established with the smooth solutions of the isothermal irrotational inviscid gas dynamics equations.

Key words. Harmonicity, gas dynamics, rearrangement, vibrating strings

\section{Review of Some Classical Concepts}

1.1. Harmonic (or Wave) Maps. Let $S>0, T>0, U=] 0, T[\times] 0, S[$ and $D=\mathbf{T}^{d}=(\mathbf{R} / \mathbf{Z})^{d}$ be the unit periodic cube. A map $(t, s) \in U \rightarrow X(t, s) \in D$ is usually called a harmonic map (resp. a wave map) if it is a critical point of the functional

$$
\int_{U} \frac{1}{2}\left(\eta\left|\partial_{t} X(t, s)\right|^{2}+\left|\partial_{s} X(t, s)\right|^{2}\right) d t d s
$$

where $\eta=1$ (resp. $\eta=-1$ ), with respect to perturbations with compact support in $U$. (Here $|$.$| denotes the Euclidean norm on \mathbf{R}^{d}$.) This means that $X$ solves the homogeneous Laplace (resp. wave) equation

$$
\eta \partial_{t t} X+\partial_{s s} X=0
$$

In the harmonic case $\eta=1$, a map $X$ is called minimizing harmonic if it minimizes (1.1) as its value $\left.X\right|_{\partial U}$ along the boundary is fixed.

REMARK. A natural class for the "target" $D$ of a harmonic (or wave) map is the class of general Riemannian manifolds. The case $D=\mathbf{T}^{d}$ is fairly trivial but sufficient for our discussion. Compact Riemmanian manifolds without boundary could also be considered. However, compact manifolds with boundaries or non compact manifolds, in particular the Euclidean space $\mathbf{R}^{d}$, would cause difficulties.

1.2. Laws, Rearrangements, Moser's Lemma. Let $(A, d a)$ be a probability space (typically $A=[0,1]$ or $A=\mathbf{T}^{d}$ equipped with the Lebesgue measure $d a$ ). For a measurable function $a \in A \rightarrow X(a) \in D$, we define the "law" of $X$ to be

$$
\mu(x)=\int_{A} \delta(x-X(a)) d a
$$

which is a probability measure on $D$, more precisely defined by

$$
\int_{D} h(x) d \mu(x)=\int_{A} h(X(a)) d a,
$$

\footnotetext{
*Received: January 25, 2002; Accepted (in revised version): May 12, 2002.

†CNRS, Laboratoire Dieudonné, Université de Nice, France (on leave from Université Paris 6), (brenier@math.unice.fr). Work partly supported by the Austrian project START (FWF-TEC-Y-137) and TICAM (University of Texas, Austin).
} 
for all $h \in C(D)$. (An usual denomination for $\mu$ is "push-forward" of $d a$ by $X$.) We now say that two such measurable functions $X$ and $Y$ are equal up to rearrangement if they have the same law, namely

$$
\int_{A} \delta(x-Y(a)) d a=\int_{A} \delta(x-X(a)) d a .
$$

Let us quote a very useful result, due to Moser and later improved by Dacorogna and Moser [DM], at least in the special case $A=\mathbf{T}^{d}$.

Lemma 1.1. Let $A=D=\mathbf{T}^{d}$. Let $\rho(x)>0$ be a smooth function of $x \in D$ with mean 1. Then there is a smooth orientation preserving diffeomorphism $X$ from $A$ to $D$ such that $\rho(x) d x$ is the law of $X$. In addition, as $\rho$ depends smoothly on some parameter $s \in[0,1]$, we may find $X$ that also depends smoothly on $s$.

An elementary proof is provided in Section 8.8

1.3. Isothermal Irrotational Gas Dynamics. The evolution of an inviscid gas moving in the Euclidean space $\mathbf{R}^{d}$ (physically $d=1,2,3$ ) is described by a density field $\rho(s, x)>0$, a pressure field $p(s, x)>0$, a temperature field $\Theta(s, x)>0$, and a velocity field $u(t, x)$, valued in $\mathbf{R}^{d}$, where $s$ stands for the time variable and $x$ for the space variable. These fields are subject to the following equations (where we use notations $\nabla$ for $\left(\partial_{x_{1}}, \ldots, \partial_{x_{d}}\right)$ and . for the Euclidean inner product in $\left.\mathbf{R}^{d}\right)$

$$
\partial_{s} \rho+\nabla \cdot(\rho u)=0,
$$

which expresses the conservation of mass and is usually called "the continuity equation,"

$$
\partial_{s} u+(u . \nabla) u+\frac{\nabla p}{\rho}=0,
$$

which is equivalent to the conservation of momentum. The evolution is called isothermal if the temperature field is a constant $\Theta(s, x)=\Theta>0$ and the pressure is given by

$$
p=\eta \rho \Theta
$$

for some constant $\eta>0$. Introducing

$$
\lambda=\sqrt{\eta \Theta} \log \rho,
$$

these equations can be written as a symmetric first-order hyperbolic system in spacetime variables $(x, s)$

$$
\begin{gathered}
\partial_{s} \lambda+u . \nabla \lambda+\sqrt{\eta \Theta} \nabla \cdot u=0, \\
\partial_{s} u+(u . \nabla) u+\sqrt{\eta \Theta} \nabla \lambda=0,
\end{gathered}
$$

for which existence and uniqueness of smooth solutions, for smooth initial data and short time intervals, are standard results (see [Si] for instance). Potential velocity fields of form

$$
u=\nabla \phi,
$$


with (scalar) potentials $\phi(s, x)$, are preserved during the evolution, as long as they stay smooth. Then, the continuity equation becomes

$$
\partial_{s} \rho+\nabla \cdot(\rho \nabla \phi)=0
$$

and the momentum equation can be integrated out, which leads (up to an irrelevant constant) to :

$$
\partial_{s} \phi+\frac{1}{2}|\nabla \phi|^{2}+\eta \Theta \log \rho=0 .
$$

Then, equations (1.13), (1.14), form a self-consistent system of evolution PDEs in $\rho$ and $\phi$, describing isothermal irrotational inviscid gas dynamics.

Remark on the Action principle. At a formal level, equations (1.13), (1.14) can be easily derived by varying the Action

$$
\int\left(\frac{|E|^{2}}{2 \rho}-\eta \Theta \rho \log \rho\right) d s d x
$$

with respect to $\rho(s, x)>0, E(s, x) \in \mathbf{R}^{d}$, subject to

$$
\partial_{s} \rho+\nabla \cdot E=0 .
$$

Indeed, $\phi(s, x)$ is introduced as a Lagrange multiplier for constraint (1.16). By varying the associated Lagrangian

$$
\int\left(\frac{|E|^{2}}{2 \rho}-\eta \Theta \rho \log \rho-\partial_{s} \phi \rho-\nabla \phi . E\right) d s d x
$$

with respect to $(\rho, E, \phi)$, we get $E=\rho \nabla \phi$ and (1.14). Of course, as usual for hyperbolic equations, the Action principle does not correspond to a minimization problem. Notice that, in the unphysical case $\eta=-1$, Action (1.15) becomes a convex functional of $(\rho, E)$. Then, the corresponding change of sign for $\log \rho$ in equation (1.14) transforms system (1.13), (1.14) into an elliptic system in space-time variables $(x, s)$.

\section{Harmonic or Wave Maps up to Rearrangement}

Let us now combine the concepts of harmonic (or wave) maps and rearrangements.

DEFINITION 2.1. We say that

$$
(t, x, a) \in U \times A \rightarrow X(t, s, a) \in D
$$

is a harmonic map (resp. a wave map) up to rearrangement, in short a $H M U R$ (resp. aWMUR), if it is a critical point of

$$
J(X)=\inf _{Y} \int_{U \times A} \frac{1}{2}\left(\eta\left|\partial_{t} X(t, s, a)\right|^{2}+\left|\partial_{s} Y(t, s, a)\right|^{2}\right) d t d s d a
$$

with respect to perturbations with compact support in $U$, where the infimum is performed over all possible rearrangement $Y$ of $X$ with respect to $a \in A$, i.e. for all functions $Y$ such that

$$
\int_{A} h(Y(t, s, a)) d a=\int_{A} h(X(t, s, a)) d a
$$


holds true for all $h \in C(D),(t, s) \in \bar{U}$. We call $J(X)$ the Dirichlet integral of $X$ "up to rearrangement" (even in the case $\eta=-1$, for simplicity).

Our main goal is to show that the equations governing the HUR maps are related to fluid mechanics and, more specifically, to the equations of isothermal irrotational inviscid gas. In order to establish this link, it is useful to introduce the "phase" density $f(t, s, x, \xi) \geq 0$ associated to a given map $X(t, s, a)$ and defined by

$$
f(t, s, x, \xi)=\int \delta(x-X(t, s, a)) \delta\left(\xi-\partial_{t} X(t, s, a)\right) d a
$$

for $(t, s, x, \xi) \in \bar{U} \times D \times \mathbf{R}^{d}$.

DEFINITION 2.2. We say that $X(t, s, a)$ is a homogeneous map if the phase density $f$ defined by (2.3) is $t$ independent and factorized

$$
f(s, x, \xi)=\rho(s, x) G(\xi),
$$

where $G>0$ is a smooth radially symmetric probability density on $\mathbf{R}^{d}$ and $\rho>0$ a probablity density on $[0, S] \times D$.

One of our main results (Theorem 5.1) will show a precise correspondence between smooth solutions of isothermal irrotational inviscid gas dynamics equations and smooth $H U R$ maps which are homogeneous in the sense of Definition 2.2. In addition, it will be shown that for most of such maps, the density $G$ must be a centered Gaussian law with uniform temperature $\Theta>0$, namely

$$
G(\xi)=(2 \pi \Theta)^{-d / 2} \exp \left(-\frac{|\xi|^{2}}{2 \Theta}\right) .
$$

2.1. A Model of Collectively Vibrating Strings. The original motivation of the concept of harmonic and wave maps up to rearrangement was the following (artificial, up to our knowledge) mechanical model of vibrating strings, which corresponds to the case $\eta=-1$. Let us consider a collection of vibrating strings parameterized by

$$
s \in[0, S] \rightarrow X(t, s, a)
$$

and labelled by $a \in A$. The kinetic energy of each vibrating string is evaluated individually

$$
\int_{0}^{S} \frac{1}{2}\left|\partial_{t} X(t, s, a)\right|^{2} d s
$$

and then integrated in $a \in A$ and $t \in[0, T]$. This leads to the first part of $J(X)$, defined by (2.1). The potential energy of $X$ is not evaluated individually for each vibrating string by the usual formula

$$
\int_{0}^{S} \frac{1}{2}\left|\partial_{s} X(t, s, a)\right|^{2} d s,
$$

but rather collectively by rearranging the labels of the strings for each fixed value of $(t, s)$, in order to get the lowest possible energy. In other words, we consider all possible collections of fictitious vibrating strings

$$
s \in[0, S] \rightarrow Y(t, s, a)
$$


having the same space repartition as $X$, i.e. satisfying (2.2), and compute in this class the infimum of

$$
\int_{A} \int_{0}^{S} \frac{1}{2}\left|\partial_{s} Y(t, s, a)\right|^{2} d s d a
$$

Integrating in $t$ the resulting infimum leads to the second part of $J(X)$. As will be shown, a $W U R$ map is homogeneous, in the sense of definition 2.2, as the strings vibrate with a collective Gaussian distribution of velocities with uniform temperature $\Theta>0$. A cruder version of this model of vibrating strings has been considered in [Br2], where the variable $s$ is discrete with two values, say 0 and 1 , and the potential energy is defined with a finite difference instead of an $s$ derivative. The corresponding model is linked to incompressible fluid mechanics (Euler equations) and plasma physics (Vlasov-Poisson equations).

\section{Evaluation of the Dirichlet integral "Up to Rearrangement"}

Our first result gives a more tractable formula for the Dirichlet integral "up to rearrangement," involving a second-order linear elliptic equation, at least in the case when the law of $X$ is a smooth function $\rho>0$.

Theorem 3.1. Let $X(t, s, a)$ be a map $\bar{U} \times A \rightarrow D$ with $D=\mathbf{T}^{d}$. Assume its law

$$
\rho(t, s, x)=\int \delta(x-X(t, s, a)) d a
$$

to be a smooth (strictly) positive function on $\bar{U} \times D$. Then,

$$
J(X)=\int_{U \times A} \frac{1}{2}\left(\eta\left|\partial_{t} X(t, s, a)\right|^{2}+\left|\partial_{s} Y(t, s, a)\right|^{2}\right) d t d s d a,
$$

where $Y$ is defined by

$$
\partial_{s} Y(t, s, a)=(\nabla \phi)(t, s, Y(t, s, a)), \quad Y(t, s=0, a)=X(t, 0, a),
$$

and for each fixed $t$ and $s, \phi(t, s, x)$ is the unique solution, with zero mean, in $x \in \mathbf{T}^{d}$, of the elliptic equation

$$
-\nabla \cdot(\rho \nabla \phi)=\partial_{s} \rho
$$

A proof is provided in Section 8.1.

\section{The HMUR and WMUR Equations}

Our second result provides the equations satisfied by smooth $H U R$ (or WUR) maps $X$ with smooth law $\rho>0$.

Theorem 4.1. Let $X(t, s, a)$ be a smooth map $\bar{U} \times A \rightarrow D$ with $D=\mathbf{T}^{d}$. Assume its law,

$$
\rho(t, s, x)=\int \delta(x-X(t, s, a)) d a,
$$

to be a smooth (strictly) positive function on $\bar{U} \times D$. Then, $X$ is HUR (resp. WUR) if and only if there are two smooth real functions $\phi$ and $q$ depending on $(t, s, x) \in \bar{U} \times D$ such that

$$
\partial_{s} \rho+\nabla \cdot(\rho \nabla \phi)=0, \quad \partial_{s} \phi+\frac{1}{2}|\nabla \phi|^{2}=q,
$$




$$
\eta \partial_{t t} X(t, s, a)=(-\nabla q)(t, s, X(t, s, a)), \quad \rho(t, s, x)=\int \delta(x-X(t, s, a)) d a,
$$

with $\eta=1$ (resp. $\eta=-1$ ). In addition, (4.3) can be expressed in terms of the phase density $f$ defined by (2.3) as

$$
\partial_{t} f+\xi . \nabla_{x} f-\eta \nabla q . \nabla_{\xi} f=0, \quad \int f(t, s, x, d \xi)=\rho(t, s, x) .
$$

The proof of Theorem 4.1 is provided in Section 8.2. Subsequently, we call $H M U R$ (resp. $W M U R$ ) equations the combination of (4.2) and either (4.3) or (4.4), for $\eta=1$ (resp. $\eta=-1$ ). The study of the initial value problem is entirely open. The coupling between equations (4.2) and (4.4) is very peculiar -both $t$ and $s$ play the role of a "time" variable, $t$ for (4.4) and $s$ for (4.2)- and, of course, highly nonlinear. However, in the special case of homogeneous solutions, in the sense of Definition 2.2, these equations can be related to classical fluid mechanics.

\section{Link between HMUR Equations and Gas Dynamics}

THEOREM 5.1. Let us consider a smooth solution $(f, \rho, \phi)$ to the HMUR (resp. $W M U R$ ) equations (4.4), (4.2), where $f$ is the phase density defined by (2.3) for some map $X$.

Assume that $\rho$ does not depend on $t$ and that, for some $\left.s_{0} \in\right] 0, S[$, there is a non degenerate maximum point $x_{0}$, i.e. $\left(\partial_{x_{i} x_{j}}^{2} \rho\left(s_{0}, x_{0}\right)\right)>0$ in the sense of symmetric matrices.

Then $X$ is a homogeneous map in the sense of Definition 2.2, if and only if

$$
f(s, x, \xi)=\rho(s, x) G(\xi),
$$

where i) $G$ is a centered Gaussian density (2.5) with uniform temperature $\Theta>0$, ii) $(\rho, \phi)$ does not depend on $t$ and solve the isothermal irrotational inviscid gas dynamics equations (1.13), (1.14) with $\eta=1$ (resp. $\eta=-1$ ).

Proof. Notice first that the nondegeneracy condition is not artificial. Indeed $\rho(s, x)=1, \phi(s, x)=0, f(s, x, \xi)=G(\xi)$ is a trivial solution for any choice of $G$, even when $G$ is not Gaussian. The proof is straightforward. From (4.4), we get

$$
G(\xi) \xi . \nabla \rho(s, x)-\eta \nabla q(t, s, x) \rho(s, x) . \nabla G(\xi)
$$

and therefore, since $\rho$ and $G$ are (strictly) positive,

$$
\xi . \nabla \log \rho(s, x)-\eta \nabla q(t, s, x) . \nabla \log G(\xi)=0 .
$$

Since $G$ is assumed to be radially symmetric, $\nabla \log G(\xi)$ is proportional to $\xi$. It follows first that

$$
\nabla \log \rho(s, x)=-\eta c \nabla \log q(t, s, x)
$$

for some constant $c$, which cannot be equal to zero, because of the nondegeneracy condition $\left(\partial_{x_{i} x_{j}}^{2} \rho\left(s_{0}, x_{0}\right)\right)>0$ which implies that the range of $\nabla \rho\left(s_{0}, x\right)$ spans $\mathbf{R}^{d}$. Thus, we have

$$
q(t, s, x)=-\eta \Theta \log \rho(s, x),
$$


up to an irrelevant additive constant, for some constant $\Theta$ distinct from 0 . Next, we get

$$
(\xi-\Theta \nabla \log G(\xi)) . \nabla \log \rho(s, x)=0
$$

Since the range of $x \rightarrow \nabla \rho\left(s_{0}, x\right)$ spans $\mathbf{R}^{d}$, we obtain

$$
\xi-\Theta \nabla \log G(\xi)=0
$$

and deduce that $G$ is the centered Gaussian density (2.5) with uniform temperature $\Theta>0$. Finally, using (4.2), we conclude that a necessary and sufficient condition for a solution $X$ to the $H M U R$ (resp. $W M U R$ ) equations to satisfy (2.4) is that $\phi$ does not depend on $t$ and solve the isothermal irrotational inviscid gas dynamics equations (1.13), (1.14) with $\eta=1$ (resp. $\eta=-1$ ).

REMARK. Somewhat surprisingly, the physical isothermal gas dynamics equations, with $\eta=1$, are linked to $H U R$ maps, not to $W U R$ maps. The $W U R$ maps correspond to isothermal gas dynamics equations with $\eta=-1$, in which case the system is unphysical and space-time elliptic (with imaginary speeds of propagation).

\section{Minimizing HUR Maps}

In the harmonic case $\eta=1$, we can provide a suf ficient condition for a smooth map $X$ to be a minimizing $H U R$ map. In addition, we can relate the corresponding minimization problem, which is certainly not convex, to a linear maximization problem with convex constraints that can be seen as its dual problem.

Theorem 6.1. Let $(X, \rho, \phi, q)$ be a smooth solution to the HMUR equations (4.2), (4.3), as in Theorem 4.1, with $\eta=1$ and $U=] 0, T[\times] 0, S\left[\right.$. Let us denote by $X_{\mid \partial U}$ the restriction of $X$ for $(t, x) \in \partial U, a \in A$. Assume

$$
4 T^{2} \sup \left\{\sum_{i=1, j=1}^{d} \partial_{x_{i} x_{j}}^{2} q(t, s, x) \xi_{i} \xi_{j}|(t, s, x) \in \bar{U} \times D,| \xi \mid \leq 1\right\}<\pi^{2}
$$

Then, $X$ is a minimizing $H U R$ map on $U \times A$. In addition, the Dirichlet integral of $X$ “up to rearrangement" $J(X)$, defined by (2.1), is equal to the following functional

$$
\begin{gathered}
\stackrel{J}{=}\left(X_{\mid \partial U}\right)=\sup \int_{\partial U \times A}\left(\theta(t, s, X(t, s, a), a) n_{t}(t, s)\right. \\
\left.\quad+\phi(t, s, X(t, s, a)) n_{s}(t, s)\right) d H^{1}(t, s) d a,
\end{gathered}
$$

where $d H^{1}$ is the Hausdorff measure along $\partial U, n=\left(n_{t}, n_{s}\right)(t, s)$ the external normal for $(t, s) \in \partial U$, and $\theta$ and $\phi$ are smooth functions depending respectively on $(t, s, x, a)$ and $(t, s, x)$ subject to the pointwise inequality

$$
\partial_{t} \theta+\frac{1}{2}|\nabla \theta|^{2}+\partial_{s} \phi+\frac{1}{2}|\nabla \phi|^{2} \leq 0
$$

A proof will be provided in Section 8.3. 


\section{Generation of Minimizing $H U R$ Maps from Isothermal Gas Dynam-} ics

Finally, we can provide a rather explicit construction of minimizing $H U R$ maps, which are homogeneous in the sense of Definition 2.2, from smooth solutions to the isothermal gas dynamics equations. Subsequently, $\eta=1$ is dropped in the notations.

TheOREM 7.1. Let $\rho(s, x)>0, \phi(s, x)$ be a smooth solution of the isothermal irrotational inviscid gas dynamics equations (1.13), (1.14) for $s \in[0, S]$ and $x \in D=\mathbf{T}^{d}$, with normalized total mass

$$
\int_{D} \rho(s, x) d x=1
$$

For each $s$, let $y \in D \rightarrow X_{0}(s, y) \in D$ be a diffeomorphism chosen (according to Moser's lemma 1.1) with law $\rho(s, x) d x$

$$
\int_{D} \delta\left(x-X_{0}(s, y)\right) d y=\rho(s, x) .
$$

Define $A=D \times[0,1]^{d+1}$ and solve, for each fixed $s$ and $a=(y, w) \in A$, the autonomous second-order $O D E$ in $t \in[0, T]$

$$
\partial_{t t} X(t, s, a)=\left(\frac{\nabla \rho}{\rho}\right)(s, X(t, s, a)),
$$

with initial conditions

$$
X(t=0, s, a)=X_{0}(s, y), \quad \partial_{t} X(t=0, s, a)=V_{0}(w),
$$

where $a=(y, w)$ and

$$
V_{0}(w)=\left(\cos \left(2 \pi w_{1}\right), \ldots ., \cos \left(2 \pi w_{d}\right)\right) \sqrt{-2 \log \left(w_{d+1}\right)} .
$$

Assume

$$
4 T^{2} \sup \left\{\sum_{i=1, j=1}^{d} \partial_{x_{i} x_{j}}^{2}(-\log \rho(s, x)) \xi_{i} \xi_{j}|0 \leq s \leq S, x \in D,| \xi \mid \leq 1\right\}<\pi^{2} .
$$

Then, $X$ is a minimizing harmonic map up to rearrangement on $U \times A$, where $U=] 0, T[\times] 0, S[$. In addition, $X$ is homogeneous in the sense of Definition 2.2.

This result also establishes an intriguing relationship between, on one side, the isothermal irrotational inviscid gas dynamics equations, which are hyperbolic and for which the Action principle does not correspond to a minimization problem (as explained in Section 1.3), and, on the other side, the minimization of the Dirichlet integral up to rearrangement for prescribed Dirichlet boundary data and the dual problem (6.2) which is a linear maximization problem with convex constraints.

Proof. Let us consider the phase density $f$ associated with $X$ by (2.3). By construction of $\left(X(0, s, a), \partial_{t} X(0, s, a)\right)$, through (7.2), (7.4), (7.5), $f$ satisfies

$$
f(t, s, x, \xi)=\rho(s, x) G(\xi), \quad G(\xi)=(2 \pi)^{-d / 2} \exp \left(-\frac{|\xi|^{2}}{2}\right)
$$


at time $t=0$. (Indeed, $\rho(s, x)$ is the law of $X_{0}(s, y)$, by construction of $X_{0}$ from Moser's lemma, and it is well known that the Gaussian law can be written

$$
G(\xi)=\int_{[0,1]^{d+1}} \delta\left(\xi-V_{0}(w)\right) d w,
$$

where $V_{0}$ is given by (7.5).) Because of (7.3), $f$ satisfies

$$
\partial_{t} f+\xi . \nabla_{x} f+\frac{\nabla \rho}{\rho} \cdot \nabla_{\xi} f=0,
$$

where $\rho$ does not depend on $t$. Since $\rho(s, x) G(\xi)$ is an obvious solution of this equation, we conclude that (7.7) is true for all $t \in[0, T]$. By setting $q=-\log \rho$, we conclude that $(X, \rho, \phi)$ solve the $H M U R$ equation (4.3), (4.2). From Theorem 6.1, we deduce that $X$ is a minimizing $H U R$ map and from Theorem 5.1 that $X$ is homogeneous in the sense of Definition 2.2. The proof of Theorem 7.1 is therefore complete.

\section{Proofs}

8.1. Proof of Theorem 3.1. Let us introduce

$$
j(\rho)=\inf _{Y} \int \frac{1}{2}\left|\partial_{s} Y(t, s, a)\right|^{2} d a d t d s,
$$

where $Y$ is subject to

$$
\int \delta(x-Y(t, s, a)) d a=\rho(t, s, x),
$$

and $\rho$ is supposed to be smooth and (strictly) positive. Let us prove

$$
j(\rho)=\int_{U \times D} \frac{1}{2} \rho(t, s, x)|\nabla \phi(t, s, x)|^{2} d t d s d x,
$$

where $\phi$ solves (3.4). Notice that the minimization problem involved in definition (8.1) requires no boundary conditions. Introduce

$$
E(t, s, x)=\int \partial_{s} Y(t, s, a) \delta(x-Y(t, s, a)) d a .
$$

Observe that this vector-valued measure $E$ is absolutely continuous with respect to $\rho$. By Jensen's inequality, its (vector-valued) density, denoted by $e(t, s, x)$, is square integrable with

$$
\int \frac{1}{2}|e(t, s, x)|^{2} \rho(t, s, x) d t d s d x \leq \int \frac{1}{2}\left|\partial_{s} Y(t, s, a)\right|^{2} d a d t d s .
$$

In addition, from (8.4), we deduce

$$
\partial_{s} \rho+\nabla \cdot(\rho e)=0 .
$$

(Indeed, for all $t \in[0, T]$ and all smooth functions $g(s, x)$ compactly supported in $0<s<S$ :

$$
\int_{U \times D}\left(\rho \partial_{s} \phi+\rho e . \nabla \phi\right) d s d x=\int_{U \times D}\left(\rho \partial_{s} \phi+E . \nabla \phi\right) d s d x
$$




$$
\left.=\int\left(\partial_{s} g+\partial_{s} Y(t, s, a) . \nabla g\right)(s, Y(t, s, a)) d s d a=\int \frac{d}{d s}(g(s, Y(t, s, a))) d s d a=0 .\right)
$$

Thus, we get a lower bound for $j(\rho)$ by minimizing the left-hand side of (8.5) in $e$, subject to (8.6). Since $\rho>0$ is fixed, the unique solution is given by $e=\nabla \phi$, where $\phi(t, s, x)$ is, for each fixed $t, s$, the unique solution, with zero mean, in $x \in \mathbf{T}^{d}$ of (3.4). So we have

$$
j(\rho) \geq \int \frac{1}{2}|\nabla \phi|^{2} \rho d t d s d x .
$$

Next, let us define $Y(t, s, a) \in D=\mathbf{T}^{d}$ for $(t, s) \in \bar{U}, a \in A$, by (3.3) and check that the law of $Y$, denoted by $\tilde{\rho}(t, s, x)$ and defined by

$$
\tilde{\rho}(t, s, x)=\int \delta(x-Y(t, s, a)) d a
$$

does not differ from $\rho$. First, $\tilde{\rho}$ solves the "continuity" equation

$$
\partial_{s} \tilde{\rho}+\nabla \cdot(\tilde{\rho} \nabla \phi)=0 .
$$

(Indeed, for all test function $g(s, x)$, vanishing at $s=0$ and $s=S$, for all fixed $t$, we have

$$
\begin{gathered}
\int\left(\partial_{s} g+\nabla \phi \cdot \nabla g\right) \tilde{\rho} d s d t=\int\left(\partial_{s} g+\partial_{s} Y(t, s, a) . \nabla g\right)(s, Y(t, s, a)) d s d a \\
\left.=\int \frac{d}{d s}(g(s, Y(t, s, a))) d a d s=0 .\right)
\end{gathered}
$$

At $s=0$, we have $Y(t, s=0, a)=X(t, 0, a)$. Thus $\tilde{\rho}(t, s, x)=\rho(t, s, x)$ at $s=0$, since $\rho$ is the law of $X$. By uniqueness of the smooth solutions to the continuity equation, we deduce that $\tilde{\rho}=\rho$ for all $s \in[0, S]$. So, $X$ and $Y$ are equal up to rearrangement with law $\rho$. It follows that

$$
j(\rho) \leq \int \frac{1}{2}\left|\partial_{s} Y(t, s, a)\right|^{2} d a d t d s
$$

(by definition (8.1))

$$
=\int \frac{1}{2} \mid \nabla \phi\left(t, s,\left.Y(t, s, a)\right|^{2} d a d t d s\right.
$$

(because of (3.3))

$$
=\int \frac{1}{2}|\nabla \phi(t, s, x)|^{2} \rho(t, s, x) d t d s d x
$$

(since $\rho$ is the law of $Y$ )

$$
\leq j(\rho)
$$

(by (8.7)), which completes the proof. 
8.2. Proof of Theorem 4.1. From Theorem 3.1, we already know that an optimal $Y$ associated with $X$ can be defined by (3.3), where $\nabla \phi$ solves the linear elliptic equation (3.4), with data depending on $\rho$. Next, going back to the original definition of $H U R$ (or $W U R$ ) maps, a necessary and sufficient condition for $X$ to be a $H U R$ (or a $W U R$ ) map is that $(X, Y, q)$ is a saddle point for the Lagrangian

$$
\int \frac{1}{2}\left(\eta\left|\partial_{t} X\right|^{2}+\left|\partial_{s} Y\right|^{2}\right) d t d s d a-\int(q(t, s, X(t, s, a))-q(t, s, Y(t, s, a))) d t d s d a,
$$

where $q(t, s, x)$ is the Lagrange multiplier for constraint (2.2). We get

$$
\begin{gathered}
\eta \partial_{t t} X(t, s, a)=(-\nabla q)(t, s, X(t, s, a)), \\
\partial_{s s} Y(t, s, a)=(+\nabla q)(t, s, Y(t, s, a)) .
\end{gathered}
$$

Therefore, $\nabla \phi$, denoted by $e$, must satisfy

$$
\partial_{s} e+(e \cdot \nabla) e=\nabla q .
$$

(Indeed, we have, for all $t \in[0, T]$ and all test function $g(s, x)$ vanishing about $s=0$ and $s=T$,

$$
\int \nabla q \rho g d s d t=\int \partial_{s s} Y(t, s, a) g(s, Y(t, s, a)) d s d a
$$

(using (8.10) and the fact that $\rho$ is the law of $Y$ )

$$
=-\int \partial_{s} Y(t, s, a) \frac{d}{d s}(g(s, Y(t, s, a))) d s d a=-\int\left(\partial_{s} g+\nabla \phi . \nabla g\right) \nabla \phi \rho d s d x
$$

(because of (3.3), and using again that $\rho$ is the law of $Y$ ).) Thus, $e=\nabla \phi$ satisfies

$$
\partial_{s}(\rho e)+\nabla \cdot(\rho e \otimes e)=\rho \nabla q
$$

and, therefore, (8.11), since $\rho>0$ satisfies (1.13).) Next, by integration of (8.10) in $x$, we get

$$
\partial_{s} \phi+\frac{1}{2}|\nabla \phi|^{2}=q,
$$

up to the addition to $q(t, s, x)$ of an irrelevant function of $(t, s)$. So, we have obtained the desired equations $(4.2),(4.3)$ for $X, \rho, \phi, q$. Finally, equation (4.4) for the phase density $f$ follows from (4.3) by a standard argument. (Indeed, for all fixed $s \in[0, S]$ and all test function $g(t, x, \xi)$ vanishing at $t=0$ and $t=T$, we have

$$
\begin{gathered}
\int\left(\partial_{t} g+\xi \cdot \nabla_{x} g-\eta \nabla q \cdot \nabla_{\xi} g\right) f d t d x d \xi \\
=\int\left(\left(\partial_{t} g\right)\left(t, X(t, s, a), \partial_{t} X(t, s, a)\right)+\partial_{t} X(t, s, a) \cdot(\nabla g)\left(t, X(t, s, a), \partial_{t} X(t, s, a)\right)\right. \\
\left.-\eta(\nabla q)(t, X(t, s, a)) \cdot\left(\nabla_{\xi} g\right)\left(t, X(t, s, a), \partial_{t} X(t, s, a)\right)\right) d t d a \\
=\int \frac{d}{d t}\left(g\left(t, X(t, s, a), \partial_{t} X(t, s, a)\right)\right) d t d a=0,
\end{gathered}
$$

since $\eta \partial_{t t} X(t, s, a)=-(\nabla q)(t, s, X(t, s, a))$.) The proof of Theorem 4.1 is now complete. 
8.3. Proof of Theorem 6.1. We use two intermediary results :

Proposition 8.1. Let $X(t, s, a)$ be a smooth function on $\bar{U} \times A$ valued in $D=\mathbf{T}^{d}$. Let $J(X)$ be defined by (2.1) as the Dirichlet integral "up to rearrangement" of $X$. Then $J(X) \geq \underline{\underline{J}}\left(X_{\mid \partial U}\right)$ where $\underline{J}\left(X_{\mid \partial U}\right)$ is defined by (6.2) as in Theorem 6.1.

Proposition 8.2. Let $X(t, s, a)$ a smooth function on $\bar{U} \times A$ valued in $D=\mathbf{T}^{d}$, and satisfying the assumptions of Theorem 6.1, including condition (6.1) on T. Then

$$
\underline{J}\left(X_{\mid \partial U}\right) \geq \frac{1}{2} \int_{U \times A}\left(\left|\partial_{t} X(t, s, a)\right|^{2}+\left|\partial_{s} Y(t, s, a)\right|^{2}\right) d t d s d a,
$$

where $\underline{\underline{J}}\left(X_{\mid \partial U}\right)$ is defined by (6.2) and $Y$ is some rearrangement of $X$ in $a \in A$.

By combining Propositions 8.1 and 8.2, we see that, under the assumptions of Proposition $8.2, X$ is a minimizing harmonic map up to rearrangement and, therefore, Theorem 6.1 follows. In addition, we get

$$
J(X)=\underline{\underline{J}}\left(X_{\mid \partial U}\right)
$$

which relates $X$ to the linear maximization problem $(6.2)$ in $(\theta, \phi)$ with convex constraint (6.3).

8.4. Proof of Proposition 8.1. Let $X(t, s, a)$ and $Y(t, s, a)$ be admissible for definition (2.1), which means that $X$ and $Y$ have the same law

$$
\rho(t, s, x)=\int \delta(x-X(t, s, a)) d a=\int \delta(x-Y(t, s, a)) d a
$$

and

$$
\left.\left.\left.\int\left(\mid \partial_{t} X(t, s, a)\right)\right|^{2}+\mid \partial_{s} Y(t, s, a)\right) \mid\right) d t d s d a<+\infty
$$

Let us introduce

$$
\begin{gathered}
c(t, s, x, a)=\delta(x-X(t, s, a)), \\
Q(t, s, x, a)=\partial_{t} X(t, s, a) \delta(x-X(t, s, a)) \\
E(t, s, x)=\int \partial_{s} Y(t, s, a) \delta(x-Y(t, s, a)) d a .
\end{gathered}
$$

As in the proof of Theorem 3.1, in Section 8.1 $E$ is absolutely continuous with respect to $\rho$ and its density, $e(t, s, x)$, satisfies

$$
\int \frac{1}{2}|e(t, s, x)|^{2} d \rho(t, s, x) \leq \int \frac{1}{2}\left|\partial_{s} Y(t, s, a)\right|^{2} d a d t d s .
$$

The left-hand side can be written as a convex function of $(\rho, e)$, because of the following (elementary) lemma (see [Br1]):

LEMMA 8.3. Let $(\sigma, m)$ be a pair of measures on some compact space $M$, respectively valued in $\mathbf{R}$ and $\mathbf{R}^{d}$. Define

$$
K(\sigma, m)=\sup \left\{\int \alpha(y) d \sigma(y)+\beta(y) \cdot d m(y), \quad \alpha(y)+\frac{1}{2}|\beta(y)|^{2} \leq 0, \quad \forall y \in M\right\},
$$


where the supremum is performed over all continuous functions on $M \alpha, \beta$, respectively valued in $\mathbf{R}$ and $\mathbf{R}^{d}$. Then $K=+\infty$ unless $\sigma$ is nonnegative, $m$ is absolutely continuous with respect to $\sigma$ with a vector-valued density $v$ which is square integrable with respect to $\sigma$. In addition, as $K<+\infty$,

$$
K(\sigma, m)=\frac{1}{2} \int|v(y)|^{2} d \sigma(y)
$$

Thus we can write (8.20) as:

$$
K(\rho, E) \leq \int \frac{1}{2}\left|\partial_{s} Y(t, s, a)\right|^{2} d t d s d a .
$$

More simply, $Q$ is also absolutely continuous with respect to $c$ and

$$
K(c, Q) \leq \int \frac{1}{2}\left|\partial_{t} X(t, s, a)\right|^{2} d t d s d a
$$

(As a matter of fact, we have an equality, but we need it not.) Let us now observe that the pairs $(c, Q)$ and $(\rho, E)$ satisfy the following integral identities for all smooth functions $\theta(t, s, x, a)$ and $\phi(t, s, x)$ :

$$
\begin{gathered}
\int_{U \times D \times A}\left(\partial_{t} \theta d c+\nabla \theta \cdot d Q\right)= \\
\int_{\partial U \times A} \theta(t, s, X(t, s, a), a) n_{t}(t, s) d H^{1}(t, s) d a \\
\int_{U \times D}\left(\partial_{s} \phi d \rho+\nabla \phi . d E\right)=\int_{\partial U \times A} \phi(t, s, X(t, s, a)) n_{s}(t, s) d H^{1}(t, s) d a
\end{gathered}
$$

(where we use that $\phi$ does not depend on $a$ and $Y$ has the same law as $X$ ). Also notice that (8.15) can be expressed by

$$
\int_{U \times D} q(t, s, x) d \rho(t, s, x)=\int_{\partial U \times A} q(t, s, x) d c(t, s, x, a)
$$

for all test function $q$. Combining all these properties, we finally deduce that

$$
J(X) \geq \underline{J}\left(\left.X\right|_{\partial U}\right)
$$

where we define

$$
\underline{J}\left(\left.X\right|_{\partial U}\right)=\inf K(c, Q)+K(\rho, E),
$$

with $(c, Q)$ and $(\rho, E)$ subject to constraints $(8.25),(8.26)$, and (8.27). Notice that these constraints only involve the values of $X(t, s, a)$ for $(t, x) \in \partial U$ which justifies our notation for $\underline{J}$. Equivalently, we can write

$$
\underline{J}\left(\left.X\right|_{\partial U}\right)=\inf _{c, Q, \rho, E} \sup _{\theta, \phi, q, \alpha, \beta, \tilde{\alpha}, \tilde{\beta}} \int\left[\tilde{\alpha}(t, s, x)-\partial_{s} \phi(t, s, x)+q(t, s, x)\right) d \rho(t, s, x)
$$




$$
\begin{gathered}
+(\tilde{\beta}(t, s, x)-\nabla \phi(t, s, x)) \cdot d E(t, s, x)]+\int\left[\left(\alpha(t, s, x, a)-\partial_{t} \theta(t, s, x, a)\right.\right. \\
-q(t, s, x)) d c(t, s, x, a)+(\beta(t, s, x, a)-\nabla \theta(t, s, x, a)) \cdot d Q(t, s, x, a)] \\
\int_{\partial U \times A}\left[\theta(t, s, X(t, s, a), a) n_{t}(t, s)+\phi(t, s, X(t, s, a)) n_{s}(t, s)\right] d H^{1}(t, s) d a
\end{gathered}
$$

where $\alpha, \beta, \tilde{\alpha}, \tilde{\beta}$ are subject to

$$
\alpha(t, s, x, a)+\frac{1}{2}|\beta(t, s, x, a)|^{2} \leq 0, \quad \tilde{\alpha}(t, s, x)+\frac{1}{2}|\tilde{\beta}(t, s, x)|^{2} \leq 0 .
$$

This is the saddle-point formulation of a convex minimization problem with convex constraints.

Proving Proposition 8.1 is now very easy. Let us set

$$
\alpha=\partial_{t} \theta+q, \quad \beta=\nabla \theta, \quad \tilde{\alpha}=\partial_{s} \phi-q, \quad \tilde{\beta}=\nabla \phi
$$

and define $q$ by (8.12) where $(\theta, \phi)$ are assumed to satisfy (6.3). This provides a bound from below for $\underline{J}\left(\left.X\right|_{\partial U}\right)$. This bound is exactly $\underline{J}\left(\left.X\right|_{\partial U}\right)$, as defined by (6.2). Thus the proof of Proposition 8.1 is complete.

Remark. By using Rockafellar's duality theorem (as stated in [Brz], Chapter 1, for instance), we could show that $\underline{J}\left(\left.X\right|_{\partial U}\right)=\underline{\underline{J}}\left(\left.X\right|_{\partial U}\right)$, which means that there is no "duality gap."

8.5. Proof of Proposition 8.2. Let $(\rho, \phi, X)$ satisfy the assumptions of Theorem 6.1. Let us first introduce $Y(t, s, a) \in D=\mathbf{T}^{d}$ defined or $(t, s) \in \bar{U}, a \in A$, by

$$
\partial_{s} Y(t, s, a)=(\nabla \phi)(t, s, Y(t, s, a)), \quad Y(t, s=0, a)=X(t, 0, a) .
$$

As in the proof of Theorem 3.1, the law of $Y$ is $\rho$. Next, introduce

$$
\theta(t, s, x, a)=\inf _{\{z(t)=x\}} \int_{0}^{t}\left(\frac{1}{2}\left|z^{\prime}(\tau)\right|^{2}-q(\tau, s, z(\tau))\right) d \tau+z(0) . \partial_{t} X(0, s, a),
$$

where the infimum is performed over all smooth paths $\tau \in[0, t] \rightarrow z(\tau) \in D$ such that $z(t)=x$. Let us state two lemmata

Lemma 8.4. The following identity is true (under the assumptions of Theorem 6.1)

$$
\begin{gathered}
\left.\int_{\partial U \times A} \phi(t, s, X(t, s, a)) n_{s}(t, s)\right) d H^{1}(t, s) d a \\
=\int \frac{1}{2}\left|\partial_{s} Y\right|^{2} d t d s d a+\int q \rho d x d t d s,
\end{gathered}
$$

where $q$ is defined by (8.12). 
Lemma 8.5. Under condition (6.1), for all $t \in[0, T]$, the infimum in definition (8.31) is achieved by a unique path $z(\tau), 0 \leq \tau \leq t$, unique solution of the two-point mixed-boundary value problem (half Dirichlet, half Neumann)

$$
z^{\prime \prime}(\tau)=(-\nabla q)(\tau, s, z(\tau)), \quad z(t)=x, \quad z^{\prime}(0)=\partial_{t} X(0, s, a) .
$$

Let us postpone the proof of Lemma 8.4, Lemma 8.5, and continue the proof of Proposition 8.2. It follows from classical theory of Hamilton-Jacobi equations (see [Ba] for instance) that, under condition $(6.1), \theta(t, s, x, a)$ is a smooth solution, for $0 \leq t \leq T$, to the HJ equation

$$
\partial_{t} \theta+\frac{1}{2}|\nabla \theta|^{2}+q=0 .
$$

Thus, by definition (8.12) of $q, \theta$ and $\phi$ satisfy constraint (8.29). In addition, using (8.33) in the special case $x=X(t, s, a)$, we see that $z(\tau)=X(\tau, s, a)$ because of $(8.9)$ and, therefore,

$$
\begin{gathered}
\theta(t, s, X(t, s, a), a)=\int_{0}^{t}\left(\frac{1}{2}\left|\partial_{t} X(\tau, s, a)\right|^{2}-q(\tau, s, X(\tau, s, a))\right) d \tau+ \\
+X(0, s, a) \cdot \partial_{t} X(0, s, a) .
\end{gathered}
$$

Differentiating (8.35) with respect to $t$ gives

$$
\partial_{t}(\theta(t, s, X(t, s, a), a))=\frac{1}{2}\left|\partial_{t} X(t, s, a)\right|^{2}-q(t, s, X(t, s, a)) .
$$

Integrating this expression with respect to $(t, s, a)$ over $U \times A$ leads to

$$
\begin{gathered}
\int_{\partial U \times A} \theta(t, s, X(t, s, a), a) n_{t}(t, s) d H^{1}(t, s) d a=\int\left(\frac{1}{2}\left|\partial_{t} X\right|^{2}-q(t, s, X)\right) d t d s d a . \\
=\int \frac{1}{2}\left|\partial_{t} X\right|^{2} d t d s d a-\int q \rho d x d t d s
\end{gathered}
$$

(since $\rho$ is the law of $X$ ). By adding this equality to identity (8.32), we have finally obtained

$$
\begin{gathered}
\int_{\partial U \times A}\left(\theta(t, s, X(t, s, a), a) n_{t}(t, s)+\phi(t, s, X(t, s, a)) n_{s}(t, s)\right) d H^{1}(t, s) d a \\
=\frac{1}{2} \int\left(\left|\partial_{t} X\right|^{2}+\left|\partial_{s} Y\right|^{2}\right) d t d s d a .
\end{gathered}
$$

From definition (6.2), we deduce (8.13) and the proof of Proposition 8.2 is completed. 
8.6. Proof of Lemma 8.4. We have

$$
\begin{gathered}
\int_{\partial U \times A} \phi(t, s, X(t, s, a)) n_{s}(t, s) d H^{1}(t, s) d a= \\
\int_{\partial U \times A} \phi(t, s, Y(t, s, a)) n_{s}(t, s) d H^{1}(t, s) d a
\end{gathered}
$$

(because $X$ and $Y$ are equal up to rearrangement)

$$
=\int_{U \times A} \partial_{s}(\phi(t, s, Y(t, s, a))) d t d s d a
$$

(by integration in $s$ )

$$
\begin{gathered}
=\int_{U \times A}\left(\partial_{s} \phi(t, s, Y(t, s, a))+\partial_{s} Y(t, s, a) . \nabla \phi(t, s, Y(t, s, a))\right) d t d s d a \\
=\int_{U \times A}\left(\partial_{s} \phi(t, s, Y(t, s, a))+|\nabla \phi(t, s, Y(t, s, a))|^{2}\right) d t d s d a
\end{gathered}
$$

(because of 8.30)

$$
=\int\left(\partial_{s} \phi+|\nabla \phi|^{2}\right) \rho d x d t d s
$$

(since $\rho$ is the law of $Y$ )

$$
=\int\left(\frac{1}{2}|\nabla \phi|^{2}+q\right) \rho d x d t d s
$$

(by definition (8.12). We also have

$$
\int \frac{1}{2}|\nabla \phi|^{2} \rho d x d t d s=\int|\nabla \phi(t, s, Y(t, s, a))|^{2} d t d s d a=\int\left|\partial_{s} Y(t, s, a)\right|^{2} d t d s d a
$$

(using (8.30)). Thus, identity (8.32) follows and the proof of Lemma 8.4 is now complete.

8.7. Proof of Lemma 8.5. Let us fix $(t, s) \in[0, T] \times[0, S], x \in D=\mathbf{T}^{d}$, $a \in A$, and define

$$
h(z)=\int_{0}^{t}\left(\frac{1}{2}\left|z^{\prime}(\tau)\right|^{2}-q(\tau, s, z(\tau))\right) d \tau+z(0) . \partial_{t} X(0, s, a),
$$

for all path $\tau \in[0, t] \rightarrow z(\tau) \in D$ such that $z(t)=x$. The second derivative of $h$ with respect to $z$ is given by

$$
D^{2} h(z)(\tilde{z}, \tilde{z})=\int_{0}^{t}\left(\left|\tilde{z}^{\prime}(\tau)\right|^{2}-\sum_{i, j=1}^{d}\left(\partial_{x_{i} x_{j}}^{2} q\right)(\tau, s, z(\tau)) \tilde{z}_{i}(\tau) \tilde{z}_{j}(\tau)\right) d \tau
$$

This quadratic form is positive definite, under condition (6.1), because of the following (modified) Poincaré inequality:

$$
\pi^{2} \int_{0}^{t}|\tilde{z}(\tau)|^{2} d \tau \leq 4 t^{2} \int_{0}^{t}\left|\tilde{z}^{\prime}(\tau)\right|^{2} d \tau
$$


which holds true for all $t>0$ and for all smooth function $\tilde{z}$ such that $\tilde{z}^{\prime}(0)=0$ and $\tilde{z}(t)=0$. (This Poincaré inequality can be established by using Fourier series, the inequality being saturated by $\tilde{z}(\tau)=\sin \left(\frac{\pi}{2 t} \tau\right)$.) It follows that condition (6.1) on $T$ is sufficient to enforce that, as long as $t \leq T$, the infimum in definition (8.31) is achieved by a unique path $z(\tau), 0 \leq \tau \leq t$, unique solution of the two-point mixed-boundary value problem (half Dirichlet, half Neumann) (8.33). Thus, the proof of Lemma 8.5 is complete.

8.8. Appendix: A Proof of Moser's Lemma. In the case where $D$ is the unit periodic cube, the proof is very easy. First solve the Laplace equation (with periodic boundary conditions)

$$
-\Delta \theta=\rho-1
$$

on $D$ (by using Fourier series, for instance). Next define

$$
v(\tau, x)=\frac{\nabla \theta(x)}{\tilde{\rho}(\tau, x)}, \quad \tilde{\rho}(\tau, x)=(1-\tau)+\tau \rho(x),
$$

for $\tau \in[0,1]$ and $x \in D$, so that

$$
\partial_{\tau} \tilde{\rho}+\nabla \cdot(\tilde{\rho} v)=0 .
$$

Then, for each $a \in D$ solve the initial value problem

$$
\partial_{\tau} Z(\tau, a)=v(\tau, Z(\tau, a)), \quad Z(0, a)=a, \quad \forall a \in A=D .
$$

Check that, for each $\tau \in[0,1], Z(t,$.$) is a smooth diffeomorphism of D$ with law $\tilde{\rho}(\tau, x) d x$. Conclude by setting $X(a)=Z(1, a)$.

Acknowledgements. The author thanks TICAM, Austin, and the Erwin Schrödinger Institut, Vienna, for their hospitality during the preparation of this work. He also thanks Luis Caffarelli and Michel Rascle for stimulating discussions.

\section{REFERENCES}

[Ba] G. Barles, Solutions de viscosité des équations d'Hamilton-Jacobi. Mathématiques et applications 17, Springer, 1994.

[Br1] Y. Brenier, Minimal geodesics on groups of volume-preserving maps. Comm. Pure Appl. Math. 52:411-452, 1999.

[Br2] Y. Brenier, Derivation of the Euler equations from a caricature of Coulomb interaction. Comm. Math. Phys. 212:93-104, 2000.

[Brz] H. Brezis, Analyse Fonctionnelle Appliquée. Masson, Paris, 1974.

[DM] B. Dacorogna and J. Moser, On a partial differential equation involving the Jacobian determinant. Ann. Inst. H. Poincar Anal. Non Linaire 7:1-26, 1990.

[Si] T.J. Sideris, The lifespan of smooth solutions to the three-dimensional compressible Euler equations and the incompressible limit. Indiana Univ. Math. J. 40:535-550, 1991. 